\title{
Phytotherapie
}

\section{Was macht Paprika bei Halsschmerzen?}

- Halsschmerzen aufgrund von Entzündungen des oberen Rachens, des Halsraumes oder der Mandeln gehören in Deutschland mit einer jährlichen Inzidenz von 30\% zu den häufigsten Symptomen einer Erkältung. In 80\% der Fälle sind Viren (Rhino-, Coronaviren) die Ursache. Hier ist die symptomatische Therapie Mittel der Wahl. Doch obwohl Halsschmerzen ein häufiger Beratungsanlass in der Praxis sind (1-2\% aller Konsultationen), greifen viele Patienten zu Beginn der Erkrankung auf

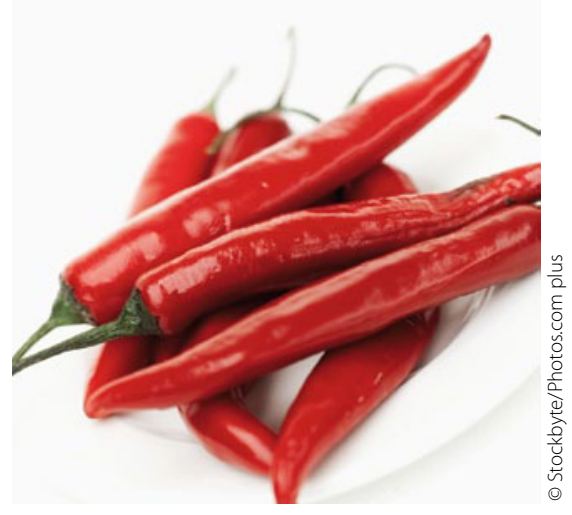

Schmerzlinderung durch den Wirkstoff Capsaicin. verschreibungsfreie Produkte aus der Apotheke zurück.

In einer apothekenbasierten vergleichenden Studie wurde Tonsipret ${ }^{\circledR}$ (Wirkstoffkombination aus Arzneipaprika, Pockholz und Kermesbeere) mit den chemisch-synthetischen Vergleichspräparaten A (Tyrothricin $4 \mathrm{mg}$, Cetrimoniumbromid 2 mg, Lidocain $1 \mathrm{mg}$ ) oder B (1,4 mg Cetylpyridiniumchlorid, $10 \mathrm{mg}$ Benzocain) verglichen. Dazu verteilten die Apotheken randomisierte Prüfbögen an Kunden, die das jeweilige Medikament ungestützt verlangt hatten, über Halsschmerzen und/oder Schluckbeschwerden klagten und älter als 18 Jahre waren.

\section{Doppelte Wirksamkeit}

Eine Zwischenauswertung (660 Fragebögen aus 213 Apotheken) belegt die doppelte Wirksamkeit des Phytotherapeutikums bezüglich Schmerzlinderung und Heilungsförderung (Jund R et al. Planta Medica 2011; 77(12):1229-1472). 76\% der Patienten berichteten über eine Schmerzlinderung innerhalb von weniger als 30 Min., die v. a. durch den Wirkstoff Capsaicin der Arzneipaprika bewirkt wird. Die schmerzlindernde Wirkung durch die Pflanzenkombination trat bei leichten bis mittleren Halsschmerzen vergleichbar schnell zum Vergleichspräparat A mit dem lokalanästhetischen Wirkstoff Lidocain und fast genauso schnell wie mit dem bei starken Halsschmerzen eingesetzten Präparat B mit Benzocain ein. Ab dem zweiten Tag des fünftägigen Beobachtungszeitraumes erreichten mehr Patienten unter dem pflanzlichen Arzneimittel Symptomfreiheit (Halsschmerzen, Schluckschmerz) als unter den beiden Lokalanästhetika. Am dritten Behandlungstag waren mit 18\% mehr als doppelt so viele Patienten komplett schmerzfrei als bei den chemischsynthetischen Präparaten (jeweils 8\%).

Auch hinsichtlich der Verträglichkeit schnitt das Phytotherapeutikum gut ab: nur $11,4 \%$ der Patienten registrierten ein Taubheitsgefühl im Hals, während diese Nebenwirkung bei den Vergleichspräparaten mit 27,5\% (Lidocain-Präparat A) bzw. $80 \%$ (Benzocain-Präparat B) berichtet wurde.

- Red.

Quelle: Nach Informationen von Bionorica SE

\section{Diabetische Polyneuropathie}

\section{Welche Therapie empfehlen die Leitlinien?}

— Etwa jeder fünfte Diabetespatient leidet an einer schmerzhaften diabetischen Polyneuropathie (DPNP). Dr. Gerhard Klausmann, Aschaffenburg, berichtete, dass zwei Drittel der mild bis mittelschwer betroffenen und ein Drittel der schwer betroffenen Patienten unentdeckt bleiben und nur ein Viertel leitliniengerecht behandelt wird. Der weitaus größere Teil erhalte vor allem nicht steroidale Antiphlogistika (NSAID) sowie Nicht-Opioid-Analgetika und Tranquilizer, die bei DPNP ungeeignet seien.

Für die Erstlinientherapie empfehlen nationale und internationale Leitlinien Duloxetin (Cymbalta $\left.{ }^{\oplus}\right)$. Die Deutsche Diabetes-Gesellschaft (DDG) empfiehlt den selektiven Serotonin-Noradrenalin-Wiederaufnahmehemmer (SSNRI) neben trizyklischen Antidepressiva (Amitriptylin) und Pregabalin als Mittel der ersten Wahl, Opioide als zweite Wahl. Das National Institute for Health and Clinical Excellence (NICE) empfiehlt Duloxetin als erste Wahl, Amitriptylin als Alternative bei Kontraindikation und Pregabalin als zweite Wahl.

Studiendaten zeigen, dass Duloxetin in den Dosierungen 60 mg bzw. 120 mg täglich verglichen mit Placebo Schmerzen schnell und effektiv reduziert und langfristig wirksam ist. Patienten, die auf $60 \mathrm{mg}$ nicht ansprechen, können von einer Steigerung der Dosis auf $120 \mathrm{mg}$ profitieren. Mit der Schmerzreduktion ist eine Verbesserung der
Alltagsfunktion assoziiert. Die schmerzlindernde Wirkung wird mit einer Verstärkung der deszendierenden Schmerzhemmung in Verbindung gebracht, so Klausmann.

Schmerztagebücher können die Therapie unterstützen. Ein alternativen Ansatz ist das Aktivitätentagebuch, in dem sich der Patient auf positive Ereignisse und Erfahrungen konzentrieren soll (www.lillypharma.de/gesundheit/diabetische-polyneuropathie/broschueren.html).

- Michael Koczorek

Quelle: Pressegespräch „Diabetes tut nicht weh - oder doch? Ansätze und Perspektiven für eine leitliniengerechte Therapie der DPNP", DDG-Jahrestagung, Leipzig, Juni 2011 (Veranstalter: Lilly) 